\title{
Assessment of Reflective and Metacognitive Practices for Electrical and Computer Engineering Undergraduates
}

\section{Dr. Samuel J Dickerson, University of Pittsburgh}

Dr. Samuel Dickerson is an assistant professor at the University of Pittsburgh Swanson School of Engineering. His general research interests lie in the area of electronics, circuits and embedded systems and in particular, technologies in those areas that have biomedical applications. He has expertise in the design and simulation of mixed-signal integrated circuits and systems that incorporate the use of both digital and analog electronics, as well as optics, microfluidics and devices that interface to the biological world. Dr. Dickerson is also interested in enhancing undergraduate engineering education, and investigates new and innovative methods for improving the learning experience for electrical and computer engineering students.

\section{Dr. Renee M Clark, University of Pittsburgh}

Renee Clark is Research Assistant Professor of Industrial Engineering and Director of Assessment for the Engineering Education Research Center (EERC) in the Swanson School of Engineering, University of Pittsburgh. She conducts research on education projects that focus on active learning and engineering professional development. Current research includes the propagation of active learning throughout the Swanson School and the use of systematic reflection and metacognitive activities within coursework. She received the Ph.D. in Industrial Engineering from the University of Pittsburgh and the MS in Mechanical Engineering from Case Western. She has over 25 years of experience as an engineer and analyst in industry and academia. She completed her post-doctoral studies in engineering education at the University of Pittsburgh.

Mr. Nan Jiang 


\section{Assessment of Reflective and Metacognitive Practices for Electrical and Computer Engineering Undergraduates}

\section{Introduction}

Reflection is thinking about what one is doing and is necessary for learning. Further, metacognition entails reflecting on one's thinking, knowing about one's knowing, and directing one's own learning. Regular reflection plays a critical role in the construction of metacognitive knowledge and self-regulatory skills, or planning, monitoring, and evaluating one's own learning and knowledge. Metacognition is important because it supports the development of lifelong learning skills needed to excel in the workforce. It provides students with strategies for when new tasks arise, as they must be able to navigate independently after college by directing their own learning. The literature has highlighted a lack of frequent, structured reflection and metacognition in the engineering curriculum as well as the need for additional scholarly work on reflection in engineering education. To this end, as part of a National Science Foundation (NSF) funded research project, we are investigating the extent to which systematic, frequent reflection enhances students' reflective and metacognitive practices in a microelectronics course.

In this course, the computer-aided-design environment SPICE (Simulation Program with Integrated Circuit Emphasis) is used. With SPICE, students and industry professionals develop and test electronic circuits and predict circuit behavior via simulations. Using SPICE for reflection after an exam or exercise can enable students to review, verify, and correct their hand calculations. Also, they can further explore the circuit's behavior and otherwise revisit their analysis prior to moving on. This encourages students to "think about their thinking and analysis" and discover areas for improvement. The PI and Co-PI recently developed and are assessing a SPICE-based approach to drive reflection and deeper conceptual understanding in this undergraduate microelectronics course. SPICE was used in a holistic manner for inductive lecturing, in-class paired activities involving simulation, and post-exam reflection.

In this paper we present preliminary results from our study involving reflection with SPICE. A pre-study survey provided a baseline of our student population, including students' definition of reflection. We also analyzed an initial post-quiz reflection in which they were instructed to reflect using the SPICE simulation environment, including consideration of How can I use this experience to improve in the future?

\section{Methods}

\subsection{Quiz and Reflection Using Simulation}

Homework assignments, quizzes, and exams structured in a similar manner to the example of Figure 1 were administered regularly throughout the course. In this paper, in addition to the presurvey responses, we also examined results from the first quiz. The initial quiz taken by the students consisted of 10 questions based on pre-requisite material for the course (linear circuit theory). The quiz problems were adapted from sample problems from the National Council of Examiners for Engineering and Surveying (NCEES) Professional Engineer licensure exam for 
electrical engineers. This material was selected as the basis for the quiz as it is regarded as standard knowledge for electrical and computer engineering students that have completed a linear circuit theory course.

The primary intent of this first quiz was to provide the students with training on how to reflect using the simulator, as it was to be used systematically throughout the semester. After completing a quiz, students were given access to their original quiz submissions, but the correct answers were not revealed to them. This was done to encourage students to revisit their work and think about the steps they went through to solve the quiz. Figure 1 illustrates our approach to promoting reflection using SPICE simulations. Thus, after students were given an assessment (e.g., quiz), they were asked to reevaluate the quiz problem and their responses using the SPICE simulation tool. At the end of the review process, students were asked to summarize their overall performance on the quiz and write responses to the following two prompts:

1) Explain the source of any differences between your quiz responses or calculations and the simulated results.

2) How can you use this comparison experience to improve in the future?

Knowledge assessments structured in a manner similar to the example shown in Figure 1 were administered systematically throughout the entire semester.

Step 1. Assessment

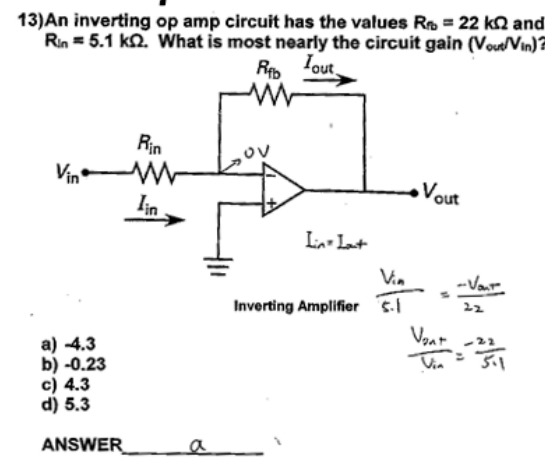

Step 2. Simulation

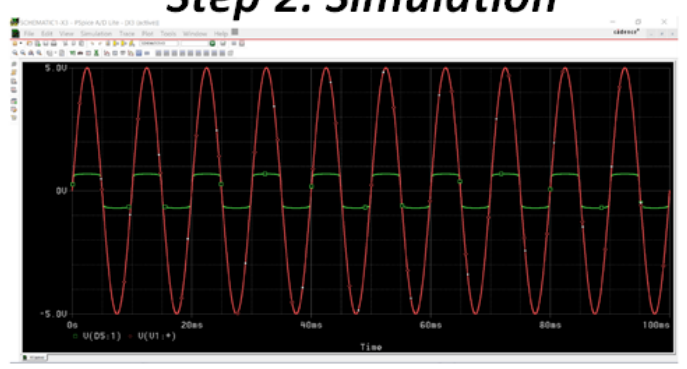

Step 3. Written Reflections

How can you use this comparison experience to improve in the future?

Figure 1. Structure of reflective simulation process.

We assessed our approach to promoting reflection by content-analyzing student responses to the second reflective questioning prompt above. After reviewing the collected responses, the coding scheme in Table 1 was developed in an emergent manner (Neuendorf, 2002). Two predominate response categories emerged, specifically responses that were found to be "broad" in nature and those that were deemed to be very "specific". Responses that were classified as being "specific" 
were those that emphasized technical aspects of the course material rather than the student's own behavior that had broader implications. For example, one student submitted the response:

"The correct answer is $C$. The voltage and current through the 5 Ohm resistor in the center is $5 \mathrm{~V}$ and $1 \mathrm{amp}$. I constructed the circuit and used bias point in the simulation"

While the student was able to correctly identify the correct answer, his/her response was focused on technical content. Broad responses were ones that had broader implications, possibly beyond the course they were in and tended to focus on the student (i.e., were behavioral-based). The actions associated with broad responses can be closely associated with the metacognitive behaviors we aim to promote (Flavell). An example "broad" response is as follows:

"Practice, practice, practice often and review often. I may remember the knowledge and feel like I am good at it, but that is not true and I still have to work harder to really understand the points and remember them in my head....Practice the new knowledge more and review the old knowledge more often will be my strategy to study in the future"

To develop the coding scheme in Table 1, we identified key phrases and indicators within the reflective responses that mapped to the two categories. Students' responses were subsequently content-analyzed based upon these two categories by two analysts independently (i.e., the assessment analyst for the project and the instructor) (Neuendorf, 2002). They subsequently discussed their codes to reach consensus on the final codes to assign.

\section{Table 1: Coding Scheme for Responses to Reflective Prompt}

\section{$\underline{\text { Broad }}$}

Need to be careful in thought processes or work

Confidence-building

Expressed aim to learn from mistakes; avoid mistakes in future

Review/look over work multiple times;

Review or reflect on work to fully understand or verify, including with simulator

Review material to refresh knowledge

Spend more time/effort studying or reviewing

Think before answering questions

\section{$\underline{\text { Specific }}$}

Better application of knowledge of circuit elements (i.e. filters, op-amps, capacitors)

Selection of correct analysis method

Need to memorize equation or definition

Highlight math errors

SPICE knowhow 


\subsection{Pre-Study Survey}

A second measure of the initial status of the students relative to their previous experience with reflection was obtained via a survey administered at the outset of the course that contained both closed and open-ended questions. The survey questions are presented in Table 2.

\section{Table 2: Pre-Study Survey Questions}

1. Do you have experience with placing reflections about your coursework or learning in writing? $(\mathrm{Yes} / \mathrm{No})$

2. Have you been asked in your other or previous courses to formally reflect on or think about your learning, performance, or achievement in the course? If so, in approximately how many courses?

3. How would you define reflection? (open ended)

\subsection{Content Analysis of Pre Survey Responses}

To assess baseline student definitions of reflection, students were asked to respond to the openended question in Table 2 on the pre-survey, "How would you define reflection?" Students' responses were content-analyzed for notable categories by two analysts (i.e., the assessment analyst for the project and a senior-level undergraduate engineering student) (Neuendorf, 2002). The coding scheme for this process was developed in an emergent manner by the analysts after reading through all responses and identifying the notable categories, which interestingly aligned with the Revised Bloom's Taxonomy, as discussed in section 2.3.1 (Krathwohl, 2002). The coding scheme consists of seven categories and is shown in Table 3.

Table 3: Coding Scheme

\begin{tabular}{l|l}
\hline \multicolumn{1}{c|}{ Code } & \multicolumn{1}{c}{ Definition or Description } \\
\hline $\begin{array}{l}\text { Look Back } \\
\text { Remember }\end{array}$ & $\begin{array}{l}\text { Retrieve information from long-term memory } \\
\text { Look back on what was previously done } \\
\text { Focus on the previous, review, remember, or re-examine the past } \\
\text { Think about something }\end{array}$ \\
\hline Understand & Understand what happened or one's thought processes \\
\hline Improve Apply & Improve or adjust for the future to better the situation \\
\hline Check & Check answers or something uncertain, especially with coursework \\
\hline Evaluate & Evaluate weaknesses, strengths, knowledge level, approaches, events, etc. \\
\hline Analyze & Break into parts and/or determine how parts are related \\
\hline Gain & Gain or create new insight \\
\hline
\end{tabular}

Using the coding scheme, each analyst independently coded the responses. They subsequently discussed their codes to assess first-time inter-rater reliability (IRR) and reach consensus on the final codes to assign. IRR is a measure that indicates the level of initial agreement with subjective analysis of qualitative data, and for this particular type of data, Cohen's kappa ( $\kappa)$ is used (Norusis, 2005). The IRR achieved by the analysts for this dataset was $\kappa=0.78$, indicating strong agreement beyond chance (Norusis, 2005). 


\subsubsection{Literature Basis for Coding Scheme}

An emergent coding scheme was developed for content-analyzing the students' responses on the pre-survey to "How would you define reflection?" The emergent coding scheme developed has similarities to previous work and perspectives in the literature on reflection. For example, Carberry et al. developed a coding scheme for student responses to the question of how they used reflection (Carberry et al., 2018). This scheme has several categories in common with the categories in the coding scheme for the present work, including Remembering and Improvement. Carberry et al. also used three categories to code definitions of reflection that align with Schon's and Rose's theories of reflection, namely "reflection on action," "reflection in action," or "reflection then action" (Carberry et al., 2018; Csavina et al., 2017). In Carberry et al.'s study, the majority of student responses (79\%) identified reflection as a means of looking back on a previous event (i.e., reflection on action), and $21 \%$ of the responses indicated that reflection was a means to impact the future by directing future choices (reflection then action) (Carberry et al., 2018). Thus, the students in this study were most likely to associate reflection with looking back on an event. In their 2014 paper identifying reflection as an area that has not been a prominent scholarly focus, Turns et al. discussed elements of reflection that also closely aligned with the categories in the present coding scheme (Turns et al., 2014). These include revisiting of an experience and stimulating recall, understanding of the experience, and action or future steps to be taken (Turns et al., 2014).

Interestingly, as the present emergent coding scheme was developed to analyze student definitions or descriptions of reflection, it began to approximately align with the hierarchical levels of the Revised Bloom's taxonomy (Krathwohl, 2002). The levels of the Revised taxonomy, in order of complexity, are as follows: 1) Remember, 2) Understanding, 3) Apply, 4) Analyze, 5) Evaluate, and 6) Create. Krathwohl defines Remember as retrieving knowledge from memory, including recalling, and he defines Understand as determining the meaning, including interpreting, summarizing, or explaining. Apply refers to implementing or carrying out a procedure, while Analyze refers to breaking something into its constituent parts and determining how the parts relate to one another or to some larger purpose. Evaluation consists of making judgments based on standards, including checking and critiquing, and Create refers to forming or generating a new product. These levels of the revised taxonomy align very closely with what emerged from the student responses to develop the coding scheme for the present work.

\section{Results}

\subsection{Pre Survey Responses}

The results of the content analysis of "How would you define reflection?" are shown in Table 4. Students most commonly identified reflection as looking back on or remembering previous events, followed by understanding of an event or thought process. Each of these were associated with over half of the student responses (67.6\%, and 52.9\%, respectively). These correspond to the lower levels of Bloom's Taxonomy, or the less-complex cognitive tasks. Interesting, Improve/Apply was the third most-frequently-occurring response (20.6\% of responses) and is the 
third level in Bloom's taxonomy. The less frequently-occurring categories of Evaluate, Check, Analyze, and Gain are each associated with the higher, more-complex levels of Bloom's Taxonomy. Evaluate and Check were separated due to the latter's focus on "checking of answers" on a test, quiz, or assignment, which was a specific leaning objective for the students as part of this research.

These results align with previous results from the literature on student free-response definitions of reflection, with Carberry et al. having found $79 \%$ of such responses associated with "looking back" (Look Back Remember) and 21\% associated with "affecting the future" (Improve Apply) (Carberry et al., 2018).

Table 4: Content Analysis Results

\begin{tabular}{l|c|c|c}
\hline \multicolumn{1}{c|}{ Code } & $\begin{array}{c}\text { Frequency of } \\
\text { Code }\end{array}$ & Responses & Percentage \\
\hline Look Back Remember & 69 & 102 & $67.6 \%$ \\
\hline Understand & 54 & 102 & $52.9 \%$ \\
\hline Improve Apply & 21 & 102 & $20.6 \%$ \\
\hline Evaluate & 17 & 102 & $16.7 \%$ \\
\hline Check & 9 & 102 & $8.8 \%$ \\
\hline Analyze & 5 & 102 & $4.9 \%$ \\
\hline Gain & 2 & 102 & $2.0 \%$ \\
\hline
\end{tabular}

These results showed that surface-level descriptions of reflection, such as "remembering" or "looking back on past actions," were the most-frequently stated definitions by students. This demonstrates an opportunity to improve the students' depth of understanding of reflection, metacognition, and the benefits. However, a notable percentage of responses did describe reflection at a deeper level, including evaluation of one's strengths and weaknesses or determining plans for future improvement.

These results corroborate findings from the closed-ended pre-survey responses and show that there is an opportunity to improve students' perception and use of reflective practices that promote metacognition. Various such questions asked students to describe their prior experience with using simulation for reflection and experiences with reflection in general in their courses. Per the pre-survey, $58 \%$ of the students did not have experience placing their reflections about their coursework in writing. Fifty percent $(50 \%)$ of the students reported that they had never been asked to reflect on their learning performance or achievement, and $31 \%$ said they had been asked to reflect in only 1 or 2 courses. These survey results revealed that the large majority of the class $(81 \%)$ had little to no experience with reflections. Thus it can be concluded that reflection was not a regular part of their usual learning strategy.

In summary, these results showed that students' prior experiences were limited; therefore, we have concluded a need to integrate these practices throughout the course and likely throughout engineering curricula in general. Based on these results showing limited experience with reflection and a surface-level perception of it, we are in the process of assessing students' 
reflective products from later in the course to determine whether their understanding of reflection and its value improved with the use of our systematic approach to reflection and metacognition.

\subsection{Results of Content Analysis of Student Reflections}

The results of the content analysis of the students' post-quiz reflections to the question "How can you use this comparison experience to improve in the future?" showed the following results. For $n=101,60 \%$ of the responses were categorized as "broad," and 54\% were deemed to be part of the "specific" category. Some responses were labeled as being both broad and specific; thus, the aforementioned percentages total a value greater than $100 \%$.

\section{Conclusions and future work}

Our pre-study assessments showed that our student population was not one that could initially be characterized as being practitioners of reflective thinking when it came to engineering topics and that they do not generally incorporate the use of reflection in their learning. In addition, students had a rudimentary, low-complexity definition of reflection at the time of the pre-survey. Despite having these characteristics, the majority of students $(60 \%, n=101)$ produced post-quiz reflective responses that were relatively more broad and metacognitive in nature. This included responses that pointed to shortcomings in their own learning-process behavior. This suggests that the simulation-based reflective exercises may have been successful in eliciting these broader, metacognitive responses, since the pre-survey responses indicated students came in to the course inexperienced in reflection and identified it with lower levels of learning.

In the next phase of the project, we will study the longer-term impact of systematic simulationbased reflective exercises used throughout the course on students' learning and ability to reflect. We hypothesize that by systematically and frequently presenting students with these exercises that we can enhance the broad "metacognitive" nature of the reflections. 


\section{References}

Carberry, A., Harding, T., Cunningham, P., Csavina, K., Ausman, M., \& Lau, D. (2018). Professional and Personal Use of Reflection by Engineering Faculty, Students, and Practitioners. Proceedings of ASEE Annual Conference and Exposition, Conference Proceedings, Salt Lake City, UT.

Csavina, K., Carberry, A., Harding, T., Cunningham, P. (2017). Work in Progress: Examining the Value of Reflection in Engineering Practice and Education. Proceedings of ASEE Annual Conference and Exposition, Conference Proceedings, Columbus, $\mathrm{OH}$.

Flavell, J. H. (1976). Metacognitive aspects of problem solving. In L. B. Resnick (Ed.), The nature of intelligence (pp.231-236). Hillsdale, NJ: Erlbaum

Krathwohl, D. (2002). A revision of Bloom's taxonomy: An overview. Theory into Practice, 41(4), 212-218.

Neuendorf, K. (2002). The Content Analysis Guidebook. Thousand Oaks, CA: Sage Publications.

Turns, J., Sattler, B., Yasuhara, K., Borgford-Parnell, J., \& Atman, C. (2014). Integrating reflection into engineering education. Proceedings of American Society of Engineering Education Annual Conference, Indianapolis, IN. 